\title{
The generative power of feature geometry*
}

\author{
András Kornai \\ CSLI, Stanford University
}

\begin{abstract}
The paper presents a formalization of the common structure underlying different proposals concerning the geometry of phonological features. Using the concepts and tools of elementary set theory, lattice theory, and the theory of independent boolean algebras, a metatheory of autosegmental feature structure is developed. This metatheory enables us to compare contemporary autosegmental phonology to the now abandoned "standard" phonology and to the historically important Pạnini-type phonology as well, and to investigate the restrictions on the use of features in phonological theory.
\end{abstract}

\section{Introduction}

Seven years after the publication of Clements (1985), the phonological literature is rife with proposals like Sagey (1986), Schein and Steriade (1986), Archangeli and Pulleyblank (ms), Halle and Ladefoged (1988) that pertain to the 'geometrical' arrangement of the features that make up phonological representations (for a survey, see McCarthy 1988). In this paper all these proposals will be taken to be structures conforming to a (largely implicit) metatheory. The main question of the paper is whether this metatheory is specific to phonology at all. To show that it is, we must demonstrate the existence of some state of affairs which can not be described by a suitable feature geometry i.e. we must put an upper bound on the generative capacity of feature geometry.

The paper investigates the ideas of autosegmental phonology embodied in features and feature geometry from a formal perspective. Some familiarity with the basic ideas of generative phonology, as can be gained from most linguistics textbooks such as Fromkin and Rodman (1974), is assumed, but no knowledge of current developments is presumed. Section 1 presents two older conceptions of features, that of SPE and that of Pānini, and shows that the modern theory of feature geometry is more general than either of these. Using the insights gained from this metatheoretical comparison, in Section 2 the generative power of feature geometry is investigated using Ehrenfeucht's theory of weakly boolean structures. Whether this generative capacity is necessary for the purposes of phonology is discussed in the concluding Section 3.

\section{What is feature geometry?}

If we wish to characterize the phonological system of a language, we need to specify the segmental inventory $S$ (defined broadly so as to include both underlying and surface phonemes) and some rules, either declarative or

\footnotetext{
*This paper benefited a great deal from the comments of the participants at MOL2, Paul Kiparsky, and an anonymous reviewer - all remaining errors are my own.
} 
procedural, which specify the mapping between underlying and surface forms. For example, the nominative form of Russian nouns can be predicted from their dative forms by removing the dative suffix $u$ and inspecting the final consonant: if it was $b$ or $p$ the final consonant of the nominative form will be $p$. This could be expressed in a phonological rule of final $b$ devoicing: $b \rightarrow p /$--\#

Most remarkably, we find that a similar rule links $d$ to $t, g$ to $k$ and in fact any voiced obstruent to its voiceless counterpart. This phenomenon, that the structural description and/or the structural change in rules will be met not only by a single segment, but rather by some bigger set of segments $R$, is in fact so pervasive that it makes a great deal of sense to introduce some formal apparatus that enables us to exploit it in our characterization of the phonological system. What is required is a clever notation that lets us characterize any such $R \subset S$, traditionally called a natural class, in a compact manner so that rules stated in terms of natural classes are just as easy, or perhaps even easier, to deal with as rules stated in terms of segments.

The set $N \subset 2^{S}$ of natural classes is not really under the control of the grammarian: it is externally given by the phonological patterning of the language. The notational devices that we use to capture natural classes are successful to the extent that they make it easier to use natural classes (i.e. members of $N$ ) than unnatural ones (i.e. those not in $N$ ) in the rules. Clearly, nothing can be won by expressing natural classes disjunctively in terms of their members, since this approach works for unnatural classes like $\{p, t, b\}$ just as well as it works for natural classes like $\{p, t, k\}$. What is needed is a more clever notation, such as the one provided by feature geometry, that exploits the internal structure of $N$ to achieve notational compactness. Since feature geometry accomplishes this goal by rather complex means, in 1.1 and 1.2 we will present two simpler formalisms aiming at compactness, and in 1.3 we show that that they are, in a well-defined sense, special cases of feature geometry. We begin in 1.1 with the well-known "standard" feature-based notation (Chomsky-Halle 1968, henceforth abbreviated SPE) and in 1.2 we discuss the perhaps less widely known, but no less interesting interval-based formalism employed by Pāninini.

\subsection{The "standard" theory}

The use of distinctive features is fundamental to both classical (SPE) and modern (autosegmental) generative phonology. But the best known formal model of features (Cherry, Halle, and Jakobson 1953) actually predates generative phonology. In brief, this model (see also Cherry 1956, Cherry 1957) describes the assignment of feature values to segments as a mapping from the set $S$ of segments into the Euclidean space of dimension $n$, where $n$ is the number of features used in the analysis. Cherry conceives of this Euclidean space as being phonetic in nature: the coordinates correspond to physically measurable properties of the sounds such as formant values.

In this paper we follow the slightly more complex technical route preferred in contemporary phonology: the direct mapping between phonological features and phonetic observables (see Stevens and Blumstein 1981) is replaced by two-stage mapping in which feature assignment is viewed as a phonemic, rather than phonetic, first step, and the resulting abstract structures are interpreted phonetically in the second step. Further, in keeping with the binary nature of phonological features, the underlying field of reals used by Cherry is replaced by the finite field $\operatorname{GF}(2) .{ }^{1}$ Thus we define a feature assignment as an injective mapping $C$ from a given set $S$ of segments $s_{1}, s_{2}, \ldots, s_{k}$ into the linear space GF(2,n). In other words, each segment is mapped on an n-tuple of 0s and 1s: at this stage of the analysis, no partially specified segments (archisegments) are permitted.

\footnotetext{
${ }^{1}$ There are only two elements in $\operatorname{GF}(2): 0$ and 1 . Arithmetic is performed in the usual way, except for the fact that $1+1=0$.
} 
As we have discussed above, a clever feature assignment must be able to capture the natural classes defined by the phonological system of the language in a notationally compact manner. Following Halle (1964:328) those classes that can be expressed by fewer features than their individual members will be called $N$-classes: in $\operatorname{GF}(2, n)$ these are the hyperplanes parallel to the axes and their set will be denoted by $\mathrm{N}(2, \mathrm{n})$. A feature assignment $C$ will be called compact if it maps sets in $N$ onto $C(S) \cap N(2, n)$. If a compact feature analysis exists, it is easy to show that the following propositions are true:

(1) The number of natural classes is small: for $|S|=k,|N| \leq k^{1.58}$

(2) The set of natural classes $\mathrm{N}$ must be basically closed under intersection: for $U, V \in N, s \in U, V$, either $U \cap V=\{s\}$ or $U \cap V \in N$

\subsection{Pāṇini's theory}

Essentially the same two propositions follow from the first extant treatment of natural classes, given in Pānini 1.1.71. Simplifying matters somewhat (for a fuller discussion, see (Staal 1962), Pāninini's method is to arrange the phonemes in a linear sequence (the śivasütras) with some indicatory letters (anubandha) interspersed. Natural classes (pratyāhāra) are defined as those subintervals of the śivasütras which end in some anubandha. The number of pratyāhāra on $k$ segments with $p$ equidistant anubandha is $\approx k(p+1) / 2 \leq k(k+1) / 2$, again a small power (at most the square) of $k$. Furthermore, the intersection of two pratyāhāra, if not empty, can also be expressed as a pratyāhāra, and is, therefore, 'natural'.

In addition to using pratyāhāra, Pānini employs a variety of other devices, most notably, the concept of 'homogeneity' (sāvarnya) as a means of cross-classification (see Cardona 1965). This idea, roughly corresponding to the autosegmental concept of a [supralaryngeal] class node, enables Pānini to treat quality distinctions in vowels separately from length, nasality, and tone distinctions, as well as to treat place of articulation distinctions in consonants separately from nasality, voicing and aspiration contrasts. Another subsidiary concept, that of antara 'nearness' is required to handle the details of mappings between natural classes. Since the rules only map classes onto classes, the image of a segment under a rule is decided by 1.1.50 sthāne 'ntaratamah 'in replacement, the nearest'.

Thus we see two rather different kinds of notations that enable the grammarian to refer not only to segments, but also to natural classes in the statement of phonological rules. In the standard theory, the savings come from the fact that only those features are mentioned which take the same value for each member of the natural class in question: for the Russian final devoicing rule mentioned above these features are [-sonorant] and [+consonantal] in the structural description, and [-voice] in the structural change of the rule. In the Panninian theory the savings come from the fact that rather than referring to the whole set of obstruents, we only have to refer to the initial member and the closing anubandha of the class. What is common to both of these theories is that their evidence comes from the phonological domain: it is the phonological clustering of segments that determines feature assignment or anubandha placement.

\subsection{Feature geometry}

Feature geometry, when formulated abstractly, turns out to be a generalization of both the standard and the Pạninian approaches. The standard theory, based on feature vectors, gives rise to the finite linear space $\mathrm{GF}(2, \mathrm{n})$ which has Boolean algebraic structure: in Section 2 this will be generalized to feature geometries using the semi-independent boolean rings (SIBRs) introduced by Ehrenfeucht (pc). As we shall see shortly, in feature geometry the linear intervals of the Pāninian model are replaced by generalized (lattice-theoretic) 
intervals, meaning that the main source of generality in feature geometry is that it permits all kinds of rooted labelnode trees, but only one of these, called the "paddlewheel" in Archangeli (1985), and the "rolodex" in Goldsmith (1990), is a notational variant of the standard model.

To establish a one-to-one correspondence between the feature vectors of the standard theory and the star-shaped trees in which all features are daughters of the root node of the tree, consider an arbitrary set of $n$ features $f_{1}, \ldots, f_{n}$. If a segment has, say, value $1(+)$ for features $f_{1}, \ldots, f_{k}$, and value $0(-)$ for $f_{k+1}, \ldots, f_{n}$, the corresponding 'geometrically arranged' feature structure will have the nodes $f_{1}, \ldots, f_{k}$, and only these, dominated by the root node. It is easy to see that the collection of feature structures corresponding to an N-class will be a collection of substars all containing some star B and contained in some larger star T. Since labeled graphs form a distributive lattice for the usual set theoretic operations of union and intersection, the elements of the N-class will thus correspond to a (closed) interval, in the lattice-theoretic sense, between B and T. Every interval of this sort will be called an M-class.

In the standard case, N-classes and M-classes coincide, and in the case of more complex geometries, Mclasses can be used to define what is meant by 'compact notation'. ${ }^{2}$ If we maintain a theory of single-valued features throughout (see e.g. van der Hulst 1988), the notion of M-classes can be used to explicate the notion of natural classes without the introduction of the coordinate vectors discussed above, but if we use two-valued features, some kind of boolean apparatus must also be used, as we shall in Section 2.

\section{Generative power}

In Section 1 we motivated the use of features by their power to express natural classes compactly and showed that feature geometry is a generalization of both the SPE and the Pạninian method of expressing such classes. But we did not motivate feature geometry itself (beyond the remark that a geometrical arrangement makes it possible to replace multi-valued features by class nodes), and we did not investigate its properties in any detail. The aim of this Section is to show what can be expressed by means of rooted labelnode trees - the question what can not be expressed by these will be discussed in Section 3 .

The standard (rolodex) geometry contains no intermediate nodes between the root and the leaves of the tree. In order to justify the generality of the feature geometry scheme we need to demonstrate the utility of such class nodes. McCarthy (1988) lists three main reasons for using class nodes: processes of assimilation, processes of reduction, and cooccurrence restrictions corresponding to autosegmental association, delinking, and OCP effects respectively. None of these phenomena can be stated on natural classes directly: assimilation involves the natural correspondence of segments in two natural classes, reduction involves the relation between a natural class and a single segment, and cooccurrence restrictions involve the use of a well-formedness condition, Leben's (1973) Obligatory Contour Principle.

The overall effect of taking these phenomena into account is to define natural classes of features (see Clements 1987). Since we already have two methods, that of SPE, and that of Pānini, to deal with natural classes, the question must be asked: why do feature geometry? Why not employ some mixture of the older methods, or some entirely new one that has nothing to do with the "geometrical" arrangement of the features? The answer provided below is that natural classes (both natural classes of features and natural classes of segments) have a particular kind of algebraic structure that makes feature geometry the appropriate tool to express natural classes. In Section 1 we have already seen some indications of the fact that natural

\footnotetext{
${ }^{2}$ Given the Linking Constraint of Hayes (1986), this definition also accords with phonological practice in the case of segments with branching root nodes like affricates and geminates, since such complex segments can never fit the same structural description or structural change as simplex segments.
} 
classes of segments have some kind of algebraic structure. First, we noted in proposition (1) that compared to the variety of classes that can be formed out of segments, only a vanishing fraction of classes are actually natural. $^{3}$ Second, we noted in proposition (2) that the set $N$ of natural classes is basically closed under intersection: if we are prepared to declare singletons and the empty set natural, the qualifier "basically" can be dropped.

Thus we have reasons to believe that there is some algebraic structure to natural classes, and that this structure is almost, but not quite, boolean: $N$ is closed under intersection, but not under complementation. Therefore it makes sense to look for some generalized boolean structure in natural classes and, as this paper argues, this is exactly what we find. First let us define, following Ehrenfeucht (pc), the notion of independence: two sets $X$ and $Y$ on some domain $D$ are independent (denoted $X \nsim Y$ ) iff none of the sets $X \backslash Y, Y \backslash X, X \cap Y, \overline{X \cup Y}$ are empty. Two sets $X$ and $Y$ are semi-independent (denoted $X \not Y$ ) iff none of the sets $X \backslash Y, Y \backslash X, X \cap Y$ are empty. Informally, independence means that no Bayesian inferences can be made: knowing that some $p \in D$ is or is not a member of $X$ gives us no information about its membership in $Y$.

Features (or classes of features) are not always (semi)independent: for instance if we know that a segment is [+low] we can infer that it is [-high]. But in the structures defined below, natural classes are not required to be (semi)independent of each other: the only requirement is that if two natural classes are (semi)independent then the sets formed from them must be also natural. Ehrenfeucht defines a set of sets $E \subset 2^{D}$ to be an independent Boolean algebra or IBA iff

(i) $X, Y \in E, X \nsim Y \Rightarrow X \cup Y, X \cap Y, X \backslash Y, Y \backslash X \in E$

(ii) $X \in E \Rightarrow \bar{X} \in E$

(iii) $\emptyset, D,\{a\}, \overline{\{a\}} \in E$ (singleton sets and their complements)

A set of sets $S \subset 2^{D}$ is a semi-independent Boolean ring or SIBR iff

(i') $X, Y \in S, X \vdash Y \Rightarrow X \cup Y, X \cap Y, X \backslash Y, Y \backslash X \in S$

(ii') $\emptyset, D,\{a\} \in S$ (singleton sets).

Can the set $N$ of natural classes, perhaps suitably extended by the empty set and singletons, play the role of the set $E$ in the above definitions? As we mentioned above, the complement of a natural class of segments need not be natural: for example, the complement of the set of mid vowels, being the set of high vowels plus low vowels plus consonants is anything but natural. Thus $N$ is not an IBA. But it is a SIBR: if two natural classes of segments are neither contained in one another nor disjoint, their union, intersection, and differences will again be natural. The same can be said about natural classes of features, at least if we restrict our attention to classes established on the basis of assimilation evidence. For if a group of features $X$ assimilates as a unit in some process $p$, and an overlapping group of features $Y$ assimilates as a unit in some process $q$, and the two groups are not distinct, the segments that fit under both ${ }^{4}$ will necessarily show

\footnotetext{
${ }^{3}$ Why sparseness is an indicator of structure is a subject we can not possibly do justice to within the bounds of this paper.

${ }^{4}$ There always be such segments as long as the processes in question are self-dual, i.e. involve both the marked and the unmarked value of the feature.
} 
both kinds of assimilation. If, on the other hand, the two sets of features are disjoint, the definition of SIBRs does not require that their union act in concert.

Before we make the argument that justifies feature geometry (as opposed to other means of classification), let us consider a few simple examples of IBAs and SIBRs. First of all, the systems of sets listed in clause (iii) and (ii') of the above definition are obviously IBAs and SIBRs respectively - let us denote them by $\mathbf{A}$. Second, traditional boolean algebras are of course IBAs and SIBRs - let us denote them by B. The third example (and the first nontrivial one) is the IBA built on $\operatorname{GF}(2,2)$ i.e. the 2-dimensional cube with points $a=(0,0), b=(0,1), c=(1,0), d=(1,1)$ by including all subsets except $\{b, c\}$ and $\{a, d\}$. As the reader can easily verify, this 14 -member set of sets is an IBA but not a SIBR. The key idea is to view these sets from the center of the square, so to speak, as segments in a cyclically ordered set of $n$ points. If all such segments are included, we get an IBA, and if we break up the circle and use linear ordering we get a SIBR. Let us denote the class of such interval structures by $\mathbf{C}$.

The argument justifying feature geometry can now based on the fact that all IBAs and SIBRs can be built from the $\mathbf{A}, \mathbf{B}$, and $\mathbf{C}$ classes of IBAs and SIBRs introduced above by arranging these in a suitable tree structure. This fact, which is Ehrenfeucht's representation theorem of IBAs and SIBRs, means that any set of natural classes (of anything) can be described by repeated application of SPE-style feature analysis (B), Pāninian type sets of intervals ${ }^{5}(\mathbf{C})$ and trivial sets $(\mathbf{A})$ as long as it has the closure properties described above. In other words, feature geometry is demonstrably sufficient for describing the structure of natural classes. But is it necessary? In the next Section we will answer this question in the affirmative.

\section{Conclusion}

In principle we can imagine that for different languages different geometrical arrangements or even different feature inventories are used. In practice, however, phonological theory posits a single, universal inventory of features and looks for a single, universally fixed geometry. Since the cross-linguistic identity of features such as [nasal] is anchored in their phonetic (acoustic and articulatory) properties, rather than in some combinatorial subtleties of their intra-language phonological patterning, this search can lead to a single object (unique up to isomorphism) that will, much like Mendeleyev's periodic table, encode a large number of regularities in a compact format. To the extent that the goal of phonological research on feature geometry is a unique object, every framework permitting a variety of geometries can be said to overgenerate.

Whether the quest for a single "substantive universal" (Chomsky 1965) structure for phonological segments will be successful remains to be seen: given the success of applying the ideas of feature geometry to sign language (see e.g. Sandler 1987), where the substantive content of the features is rather different from that found in spoken language phonology, it is perhaps unrealistic to hope for the eventual discovery of a single, genetically encoded structure. Be it as it may, in a formal analysis the long-term goals of phonological research can not be used as a substitute for its actual accomplishments. Currently there is no single generally accepted feature geometry even in the spoken language domain: rather, this is a very active field of research with a broad range of competing proposals (see e.g. Itô et al 1991). Since feature geometry research is constrained only by the metatheory made explicit in the first two sections of this paper, the question whether this metatheory is too permissive is worth exploring in greater detail.

\footnotetext{
${ }^{5}$ To see that such sets form SIBRs, consider two intervals $[A B]$ and $[C D]$. If they are are semi-independent, their intersection is non-empty, so there is a segment $x$ such that $x \leq B$ and $C \leq x$. Therefore, $C<B$ and by similar appeals to the nonemptiness of $[A B] \backslash[C D]$ and $[C D] \backslash[A B]$ it follows that $A \leq C \leq B \leq D$, and thus $[A C),(B D]$, and $[A D]$ are also intervals. (The open intervals can be replaced by closed ones because there are only finitely many points involved.)
} 
As we have seen in Section 1 above, feature geometry is a generalization of both the SPE and the Pạninian method of specifying natural classes. When it comes to natural classes of features, the first question to ask is whether these older methods, or perhaps some combination of them, are sufficient for the task. Instead of arranging features in a tree structure, could we perhaps define some metafeatures of features? Or should we perhaps subject the feature set to Pāninian analysis, defining the appropriate metaśivasūtras for features? To show that these methods are insufficient, we have to find a set of classes that resists analysis in a purely feature-based or in a purely anubandha-based framework.

Laryngeal features vs. place features vs. [continuant] vs. [nasal] (that is, the groups of features immediately dominated by [root] in McCarthy's analysis) provide exactly such an example. It is trivial to devise a system of metafeatures that would distinguish these four classes of features. But metafeatures would predict that some two-member combination of these four classes, such as [place]+[nasal] or [place]+[continuant] or [place] + [laryngeal] must itself be a natural class of features, and there is no evidence in favor of such a view. A similar argument against metaśivasūtras is harder to make at the root of the tree ${ }^{6}$, but could be made lower down the tree, e.g. for vowel features. Thus we can safely conclude that neither features nor anubandhas are sufficient to deal with the full complexity of the feature classification problem by themselves: we need some combination of these, or perhaps some entirely different classification scheme, such as the decision trees now commonly employed in speech recognition (see Bahl et al 1991).

While we cannot fully anticipate the schemes that might be proposed in future phonological work, we can gain a little more insight by comparing SIBRs to IBAs and by looking at the feature geometries that were proposed so far from the perspective of SIBRs. Ehrenfeucht's representation theorem implies that the only interesting class of IBAs that are not SIBRs will be the class of cyclic interval systems. Since such systems are outside the descriptive power of feature geometry, we can say that the metatheory of feature geometry already puts a nontrivial upper bound on the complexity of the structure of segments: it predicts that no set of phonological features can ever be arranged in a cyclic ordering in such a manner that we find (assimilatory, reduction, or cooccurrence) evidence for the grouping of those features that form arcs of the cycle. According to the formalization presented here, this lack of cyclic structures is a consequence of the broader observation that (geometrically arranged) feature sets are not closed under complementation: the complement of a set of features dominated by a single class node is not expected to be dominated by some other class node.

Turning to the extant set of proposals (a partial list was given at the beginning of the paper) we notice that the class $\mathbf{C}$ is absent from them. At the root node, all existing proposals have $\mathbf{A}$ structure, and at lower nodes, $\mathbf{A}$ or $\mathbf{B}$. Here the lack of $\mathbf{C}$ structures can not be explained by lack of closure under complementation, for Pāninian type sets of intervals are SIBRs which do not require such closure. If we take the tentative suggestion advanced in fn. 6 about major class features seriously, we can conclude that feature geometry makes full use of the range of constructions necessary for generating every SIBR, i.e. that it is strictly equivalent to SIBRs. If, on the other hand, we restrict ourselves to proposals made elsewhere, it follows that feature geometry generates only a proper subset of SIBRs. Either way, the exclusion of (cyclic) interval structures demonstrates that feature geometry does not have unlimited generative power.

\footnotetext{
${ }^{6}$ In fact a review of the relevant phonological literature suggests that for the set of major class features a Pāninian analysis is quite appropriate. The necessary linear ordering is provided by the traditional sonority hierarchy (Grammont 1895, Jespersen 1904): if major classes are arranged in order of increasing sonority, we need only one anubandha (at the end) to express sonority-based generalizations such as the cross-linguistic variety of classes that can appear as syllabic nuclei.
} 


\section{References}

[1] Diana Archangeli and Douglas Pulleyblank. 1989. The content and structure of phonological representations. ms, forthcoming from MIT Press, Cambridge MA.

[2] Diana Archangeli. 1985. Yokuts harmony: evidence for coplanar representation in nonlinear phonology. Linguistic Inquiry, 16:335-372.

[3] L.R. Bahl, P.V. de Souza, P.S. Gopalakrishnan, D. Nahamoo, and M.A. Picheny. 1991. Decision trees for phonological rules in continuous speech. ICASSP-91, pages 185-188.

[4] George Cardona. 1965. On Pāṇini's morphophonemic principles. Language, 41:225-237.

[5] Colin Cherry, Morris Halle, and Roman Jakobson. 1953. Toward the logical description of languages in their phonemic aspect. Language, 29:34-46.

[6] Colin Cherry. 1956. Roman jakobson's distinctive features as the normal coordinates of a language. In Morris Halle, editor, For Roman Jakobson. Mouton, The Hague.

[7] Colin Cherry. 1957. On human communication. MIT Press, Cambridge.

[8] Noam Chomsky and Morris Halle. 1968. The Sound Pattern of English. Harper Row, New York.

[9] Noam Chomsky. 1965. Aspects of the theory of syntax. MIT Press, Cambridge MA.

[10] George N. Clements. 1985. The geometry of phonological features. Phonology Yearbook, 2:225-252.

[11] George N. Clements. 1987. Phonological feature representation and the description of. In Anna Bosch, Barbara Need, and Eric Schiller, editors, CLS Parasession on autosegmental and metrical phonology intrusive stops, volume 23, pages 29-50.

[12] Andrzej Ehrenfeucht. 1985. (personal communication).

[13] Victoria A. Fromkin and Robert Rodman. 1974. An introduction to language. Holt, Rinehart and Winston, New York.

[14] John A. Goldsmith. 1990. Autosegmental and metrical phonology. Basil Blackwell, Cambridge MA.

[15] M. Grammont. 1895. La dissimilation consonantique dans les languages indo-euro-p'eennes et dans les languages romanes.

[16] Morris Halle and Peter Ladefoged. 1988. Some major features of the international phonetic alphabet. Language, 64:577-582.

[17] Morris Halle. 1964. On the bases of phonology. In Jerry A. Fodor and J. Katz, editors, The structure of language, pages 324-333. Prentice-Hall, Englewood Cliffs.

[18] Bruce Hayes. 1986. Inalterability in CV phonology. Language, 62:321-351.

[19] 1991. Properties of feature organization. Reading materials for NSF-sponsored workshop BNS-9021357, LSA linguistic institute, UC Santa Cruz.

[20] Otto Jespersen. 1904. Lehrbuch der Phonetik. B.G. Teubner, Leipzig. 
[21] William Leben. 1973. Suprasegmental phonology. Ph.D. Thesis, MIT, Cambridge MA. Published, New York: Garland, 1979.

[22] John J. McCarthy. 1988. Feature geometry and dependency: a review. Phonetica, 45(2-4):84-108.

[23] Elizabeth Caroline Sagey. 1986. The representation of features and relations in non-linear phonology. MIT.

[24] Wendy Sandler. 1987. Sequentiality and simultaneity in American Sign Language phonology. Ph.D. Thesis, The University of Texas at Austin.

[25] Barry Schein and Donca Steriade. 1986. On geminates. LI, 17:691-744.

[26] J. F Staal. 1962. A method of linguistic description: the order of consonants according to Pāninini. Language, 38:1-10.

[27] Kenneth N. Stevens and Sheila E. Blumstein. 1981. The search for invariant acoustic correlates of phonetic features. In P. Eimas and J. Miller, editors, Perspectives on the study of speech. Lawrence Erlebaum Associates, Hillsdale, NJ.

[28] Harry van der Hulst. 1988. The geometry of vocalic features. Leiden papers in linguistics and phonetics. 